\title{
El programa RADAR para la prevención del suicidio en adolescentes de la región de Aysén, Chile: resultados preliminares
}

\author{
RADAR: a program for the prevention of suicide in adolescents in the region of Aysen, \\ Chile, preliminary results
}

\author{
Francisco Bustamante $^{\mathrm{a}, \mathrm{b}, \mathrm{c}}$, Cinthya Urquidi ${ }^{\mathrm{a}, \mathrm{d}}$, Ramón Florenzano ${ }^{\mathrm{a}, \mathrm{b}}$, Carolina Barrueto ${ }^{\mathrm{b}, \mathrm{i}, \mathrm{c}}$, \\ Jaime de los Hoyos ${ }^{\mathrm{e}}$, Karla Ampuero ${ }^{\mathrm{b}, \mathrm{j}}$, Laura Terán ${ }^{\mathrm{f}}$, María Inés Figueroa ${ }^{\mathrm{k}}$, \\ Magdalena Farías ${ }^{\mathrm{g}}$, María Livia Rueda ${ }^{\mathrm{h}, \mathrm{l}}$, Eduardo Giacaman ${ }^{\mathrm{m}}$
}

\author{
aFacultad de Medicina, Universidad de los Andes. Santiago, Chile. \\ bServicio de Salud Mental, Clínica Universidad de los Andes. Santiago, Chile \\ 'Grupo DBT, Chile \\ dDepartamento Salud Pública y Epidemiología, Universidad de los Andes. Santiago, Chile. \\ eDirección de Informática Clínica, Red de Salud UC-CHRISTUS. Santiago, Chile. \\ fUnidad de Psiquiatría, Hospital Regional Coyhaique. Aysén, Chile \\ `Servicio de Psiquiatría, Hospital de Puerto Aysén. Aysén, Chile \\ hSub departamento de Gestión y Desarrollo de la Red, Servicio Salud Aysén. Aysén Chile \\ iPsicóloga, Magíster en Psicología Clínica \\ 'Enfermera, Magíster en Gestión y Administración en Salud \\ ${ }^{k}$ Asistente social, Coordinadora de RADAR \\ 'Matrona, Encargada del GES Salud Mental y Suicidalidad \\ mEnfermero, PhD. en Salud Mental
}

Recibido el 10 de octubre de 2017; Aceptado el 4 de diciembre de 2017

\begin{abstract}
Resumen
Objetivo: Dar a conocer los resultados preliminares del programa comunitario RADAR (Red para la Atención y Derivación de Adolescentes en Riesgo suicida). Método: Durante el 2016, RADAR fue implementado como prueba de concepto en dos colegios de Puerto Aysén de la Región de Aysén, Chile. Se capacitó un total de 409 participantes (entre alumnos, profesores de los colegios, apoderados y profesionales de la salud) para la pesquisa y derivación de adolescentes en riego de suicidio. Resultados: De un total de 144 alumnos que pasaron los sistemas de pesquisa de RADAR, se detectaron 29 casos en riesgo suicida (20\%) y 27 fueron derivados oportunamente al Servicio de Urgencia del Hospital de Puerto Aysén. En la segunda campaña de pesquisa de RADAR, 3 meses después, el 90\% de los casos ya no presentaba riesgo suicida. Conclusión: Estos resultados muestran la alta proporción de adolescentes en riesgo suicida que no son visibilizados por el sistema de salud y la factibilidad de implementar RADAR en la comunidad como una intervención preventiva efectiva.
\end{abstract}

Palabras clave: Prevención; suicidio; adolescentes 


\section{Abstract}

Objective: we present the preliminary results of the implementation of RADAR: a community suicide prevention program in adolescents implemented in two high schools in a south region of Chile. Method: In a pilot study, during 2016, we implemented RADAR in two high schools of Puerto Aysen, in in the Region of Aysen of Chile. A total of 409 actors were trained (among students, school teachers, caregivers and health professionals) for the screening and referral of high suicide risk adolescents. Results: Out of a total of 144 students who passed the RADAR screening systems, 29 cases were detected as suicide risk (20\%) and 27 of them were opportunely referred to the Emergency Service of the Hospital of Puerto Aysen. In the second RADAR screening campaign, 3 months later, $90 \%$ of the cases no longer presented suicide risk. Conclusion: These results show the high proportion of adolescents at risk of suicide who are not visible by the health system and the feasibility of implementing RADAR in the community as an effective suicide prevention intervention.

\section{Keywords:}

Prevention;

suicide;

adolescents

\section{Introducción}

Las muertes por suicidio han aumentado en adolescentes de Chile, esto posiciona al país con la tasa de suicidios más alta de Latinoamérica y la $\mathrm{OECD}^{1,2}$. La distribución de las tasas no es homogénea, las regiones del sur presentan una tasa mayor, siendo Aysén (34 por 100.000 habitantes), Los Ríos (25 por 100.000 habitantes) y Los Lagos (19 por 100.000 habitantes ) las que poseen las cifras más elevadas ${ }^{2,3}$.

Desde el año 2009 un equipo de investigadores de la Universidad de los Andes ha estado trabajando en conjunto con la población de la región de Aysén para disminuir la tasa de suicidios en jóvenes. La primera intervención fue realizar un levantamiento de información a través de reuniones con profesores y alumnos de distintos establecimientos educacionales, de salud, entidades municipales y otros organismos públicos para conocer los factores que impiden una prevención eficaz. Producto de este trabajo se identificaron cuatro problemas principales: 1) los adolescentes en riesgo no se encuentran en el sistema de salud ni tampoco consultan; 2) los profesores carecen de herramientas para pesquisa y manejo de adolescentes con problemas emocionales y riesgo suicida; 3 ) la ausencia de una red comunal para el manejo de adolescentes en riesgo y 4) la necesidad del personal de salud de contar con herramientas específicas para atender estos adolescentes.

En una segunda etapa se revisó la evidencia científica buscando intervenciones para prevenir el suicidio en adolescentes. El resultado fue que tres intervenciones específicas: -psicoeducación, la pesquisa de riesgo y la presencia de gatekeepers en los colegios-ayudan a disminuir significativamente el riesgo suicida. Los gatekeepers son representantes del colegio que reciben un entrenamiento especial para identificar y asistir adolescentes en riesgo suicida ${ }^{3,4}$. Se decidió construir un programa de prevención que incluyera estos elementos además de otro tipo de intervenciones que respondie- ran a la demanda local, tales como el funcionamiento en red (colegios y dispositivos de salud), y la generación de un modelo de atención específico para adolescentes en los servicios de urgencia. Se utilizaron como marco de referencia las recomendaciones de la Organización Mundial de la Salud (OMS) $)^{5,6}$ y del Programa Nacional de Prevención de Suicidio (PNPS) de Chile del Ministerio de Salud 7 .

El resultado fue el programa RADAR (Red para la Atención y Derivación de Adolescentes en Riesgo suicida), cuya finalidad es la pesquisa de adolescentes en riesgo y su rápida derivación a un servicio de urgencias a través de una red comunal. Para ello, RADAR cuenta con los siguientes componentes: 1) capacitación a la comunidad escolar en la detección y manejo de adolescentes en riesgo a través de un gatekeeper; 2) derivación de los casos detectados al servicio de urgencias dentro de 24 horas y al psiquiatra dentro de 1-7 días según una escala de triage de riesgo; 3 ) aplicación de instrumentos de pesquisa de riesgo suicida cada 3-6 meses; 4) capacitación al personal de salud de los servicios de urgencia en una atención protocolizada específica y 5) coordinación del funcionamiento de todos los componentes y seguimiento de los casos detectados por parte de un coordinador general de la red.

La presente comunicación tiene como objetivo dar a conocer los resultados preliminares de la implementación del programa RADAR como prueba de concepto la Región de Aysén, Chile.

\section{Material y Métodos}

Con financiamiento de Corfo de innovación social Aysén (proyecto 15IS-46638), se implementó RADAR en 2 colegios de la ciudad de Puerto Aysén, Región de Aysén, durante el año 2016.

Se capacitaron un total de 409 personas: 188 $(89,9 \%)$ alumnos, $76(36,9 \%)$ apoderados, $58(70,7 \%)$ 
profesores/administrativos de los colegios, el personal de salud ( $\mathrm{n}=34$ ) del Servicio de Urgencia del Hospital de Puerto Aysén (HPA) y representantes de otros organismos comunales $(\mathrm{n}=53)$.

Se invitó a los alumnos de I a IV medio (15 a 18 años) a contestar una encuesta online con instrumentos para pesquisa de riesgo suicida con los siguientes instrumentos: 1) la escala breve para adolescente “CCómo es tu familia?” validado por la Organización Panamericana de la Salud para países de Latino América $^{8}$; 2) la escala de Autoconcepto del adolescente de Piers Harris'; 3) la escala de Capacidades y Dificultades (SDQ-cas) en su versión para adolescentes ${ }^{10} ; 4$ ) la escala de Suicidalidad de Okasha ${ }^{11}$; 5) el cuestionario de Conductas de Riesgo de Adolescentes (ECRA) y la escala de Calidad de Vida con respecto a la salud (EQ$5 \mathrm{D}-5 \mathrm{~L})^{12}$. Además, se agregaron dos preguntas libres de anclaje para riesgo inminente de suicidio. En base a la escala de Okasha y a las dos preguntas libres se programó un algoritmo de respuestas que activan un sistema de alarma de riesgo suicida la cual notifica en tiempo real al coordinador general de RADAR, quien notifica al gatekeeper del colegio.

El protocolo de RADAR y los formularios de consentimiento y asentimiento fueron revisados y aprobados por el Comité Ético Científico del Servicio de Salud de Aysén y de la Universidad de los Andes.

\section{Resultados}

De un total de 144 alumnos $(67,9 \%)$ que pasaron por los sistemas de pesquisa, se detectaron 29 casos en riesgo suicida (20\%), de los cuales el $91 \%$ no estaba en tratamiento. 27 de ellos fueron derivados al Servicio de Urgencia del Hospital de Puerto Aysén (HPA) dentro de las $24 \mathrm{~h}$; 21 de ellos (75\%) fueron derivados al psiquiatra del HPA según el protocolo (1 a 7 días según triage) y el resto de los alumnos (8 casos) fueron atendidos en el sistema de salud privado. Con respecto a la evaluación del médico general de urgencia, en el 80\% de los casos el triage en riesgo leve, moderado o severo concordó con el diagnóstico realizado por la entrevista psiquiátrica.

Del total de alumnos detectado en la primera campaña de pesquisa de RADAR (22 casos), el 90\% de los casos ya no presentaba riesgo suicida en la segunda campaña de pesquisa activa ( 3 meses después).

\section{Discusión}

Esta comunicación breve muestra que RADAR es una estrategia preventiva selectiva e indicada, factible de ser implementada en la comunidad, para la preven- ción de suicidio en adolescentes. Según recomendaciones de la OMS, la prevención selectiva debe estar dirigida a grupos etarios más vulnerables y las prevención indicada a individuos con señales prematuras de suicidio, esto quiere decir: aquellos con ideación y/o intento previo de suicidio ${ }^{6,13}$. Bajo esta misma directriz, RADAR complementa los objetivos del PNPS ${ }^{7}$ a través de la optimización de recursos locales disponibles para un acceso oportuno a la atención en salud, la capacitación a diferentes actores (ámbito escolar, salud, familiar y la comunidad), e incorpora un nuevo actor, el coordinador de red, y un sistema de pesquisa en base a factores de riesgo conocidos. Si bien, el rol del coordinador de red y la reorganización de los procesos y tiempos fueron fundamentales; las mesas de trabajo y diálogos con las autoridades locales y regionales fueron vitales para su implementación.

RADAR, a diferencia del $\mathrm{PNPS}^{7}$, incluye dos sistemas de pesquisa, uno activo y otro pasivo, cuyos resultados preliminares muestran la alta proporción de adolescentes en riesgo suicida que no son visibilizados por el sistema de salud. Por otro lado, los cuestionarios usados en el sistema de pesquisa activa prometen ser estrategias costo efectivas para prevención como se ha demostrado en otras áreas, por ejemplo en la prevención de enfermedades crónicas no transmisibles ${ }^{14}$. En este sentido, RADAR incluyó una escala que aborda la suicidalidad de forma directa la cual está validada en Chile $^{11}$. De igual manera, basado en la efectividad de la capacitación de gatekeepers, la inclusión de estos en RADAR y en los colegios piloto, permitieron la pesquisa y derivación de estos adolescentes ${ }^{4}$.

Otro hallazgo interesante fue la alta concordancia del sistema de triage usado en RADAR con la entrevista psiquiátrica. La adaptación del triage del PNPS al contexto del servicio de urgencia del hospital local, permitió optimizar los recursos humanos y logísticos disponibles para la atención de los adolescentes pesquisados. Todos los adolescentes pesquisados fueron, derivados, atendidos y tratados por el sistema de salud local, y solo una minoría dio un resultado positivo en la segunda ronda de pesquisa, 3 meses después. Finalmente, durante la implantación de RADAR, no se registraron intentos de suicidio en adolescentes en Puerto Aysén.

Los resultados preliminares en el primer año de implementación de RADAR son consistentes con los reportados por la evidencia. Una reciente revisión sistemática de 165 estudios de prevención de suicidio, indica que los programas de intervención en colegios disminuyen significativamente la conducta suicida en adolescentes, es decir el intento y la ideación suicida ${ }^{15}$. No obstante, es necesario la validación de RADAR a mayor escala y la evaluación prospectiva de su impacto. 


\section{Responsabilidades éticas}

Protección de personas y animales: Los autores declaran que los procedimientos seguidos se conformaron a las normas éticas del comité de experimentación humana responsable y de acuerdo con la Asociación Médica Mundial y la Declaración de Helsinki.

Confidencialidad de los datos: Los autores declaran que han seguido los protocolos de su centro de trabajo sobre la publicación de datos de pacientes.

Derecho a la privacidad y consentimiento informado: Los autores han obtenido el consentimiento informado de los pacientes y/o sujetos referidos en el artículo. Este documento obra en poder del autor de correspondencia.

\section{Financiación}

Proyecto financiado por CORFO para la Innovación Social Aysén (proyecto 15IS-46638)

\section{Conflicto de intereses}

Los autores declaran no tener conflicto de intereses.

\section{Agradecimientos}

Agradecemos al Dr. David L. Streiner del Departamento de Psiquiatría de la Universidad de Toronto.

\section{Referencias}

1. OECD. "Suicides", in OECD Factbook 2013: Economic, Environmental and Social Statistics [Internet]. 2013. Available from: http://www.oecd-ilibrary.org/ docserver/download/3012021ec097.pdf?ex pires $=1470709359 \&$ id $=$ id\&accname $=$ gues t\&checksum $=1 \mathrm{C} 32 \mathrm{ACDF} 5 \mathrm{~B} 39 \mathrm{FAD} 2 \mathrm{~B} 80 \mathrm{E}$ E295C680C12C.

2. Bustamante F, Ramírez V, Urquidi C, Bustos V, Yaseen Z, Galynker I. Trends and Most Frequent Methods of Suicide in Chile Between 2001 and 2010. Crisis. 2016;37(1):21-30.

3. Bustamante VF, Florenzano U R. Programas de prevención del suicidio adolescente en establecimientos escolares: una revisión de la literatura. Rev Chil Neuro-Psiquiatr. 2013;51(2):126-36.

4. Isaac M, Elias B, Katz LY, et al. Gatekeeper Training as a Preventative Intervention for Suicide: A Systematic Review. Can J Psychiatry. 2009;54(4):260-8.

5. WHO Europe. For which strategies of suicide prevention is there evidence of effectiveness? [Internet]. [citado el 4 de mayo de 2013]. Available from: http:// www.euro.who.int/_data/assets/pdf_ file/0010/74692/E83583.pdf

6. WHO | Preventing suicide: A global imperative [Internet]. WHO. 2013 [citado el 24 de abril de 2016]. Available from: http://www.who.int/mental_health/ suicide-prevention/world_report_2014/ en/

7. Ministerio de Salud. Programa Nacional de Prevención del Suicidio. 2013.

8. Organización Panamericana de la Salud. Familia y adolescencia: indicadores de salud. Manual de aplicación de instrumentos. Washington DC: Organización Panamericana de la Salud; $1996.51 \mathrm{p}$.

9. Gorostegui M, Dörr A. La escala de evaluación del autoconcepto para niños, de Piers Harris : actualización de normas. Rev Psicol Acad [Internet]. 2004;(7). Available from: http://bibliotecadigital. academia.cl/handle/123456789/2442.

10. GOODMAN R. Psychometric Properties of the Strengths and Difficulties Questionnaire. J Am Acad Child Adolesc Psychiatry. 2001;40(11):1337-45.
11. Salvo G L, Melipillán A R, Castro S A. Confiabilidad, validez y punto de corte para escala de screening de suicidalidad en adolescentes. Rev Chil Neuro-Psiquiatr. 2009;47(1):16-23.

12. Szende A, Bas J, Cabases J. Self-reported population health: an international perspective based on EQ-5D [Internet]. 2014th ed. Netherlands: Springer; Available from: https://link.springer.com/ content/pdf/10.1007/978-94-007-7596-1. pdf

13. Steele IH, Thrower N, Noroian P, Saleh FM. Understanding Suicide Across the Lifespan: A United States Perspective of Suicide Risk Factors, Assessment \& Management. J Forensic Sci. 2017; n/a-n/a.

14. Gillett M, Brennan A, Watson P, et al. The cost-effectiveness of testing strategies for type 2 diabetes: a modelling study. Health Technol Assess Winch Engl. 2015;19(33):1-80.

15. Zalsman G, Hawton K, Wasserman D, et al. Suicide prevention strategies revisited: 10-year systematic review. Lancet Psychiatry. 2016;3(7):646-59. 